\title{
ET QUELQUES VOIX DE PLUS: DE FRANCISCO NÚÑEZ MULEY À FATIMA RATAL
}

\author{
Bernard Vincent*
}

Que n'a t-on lu ou entendu à propos de la "nation" mudejar et morisque? Il a été dit et répété à satiété qu'elle était une communauté sans cesse soumise à la méfiance, au soupçon ou à la haine de la société majoritaire et donc vouée au repli, au silence ou à la clandestinité. Dés lors, il semblait que pour tenter de cerner ses activités ou/et ses aspirations, nous étions contraints de nous en remettre aux discours des autres, de l'Autre chrétien, il est vrai intarissable en la matière. Et si les spécialistes de la littératura aljamiada ont découvert un nombre non négligeable de manuscrits où les minoritaires s'expriment, longtemps nous avons cru que cet apport était limité à deux époques, celle mudejar antérieure à 1525 et celle de la diaspora, postérieure à 1609. Nous savons aujourd'hui que sa production a été continué. Mais en dehors de ce filon, les possibilités de donner la parole aux musulmans ou crypto-musulmans soumis au joug chrétien ont semblé minces.

Dans ces conditions proposer pour thème d'une rencontre "la voix des mudejars et des morisques" était une gageure. Pourtant au terme des trois journées de travail nous pouvons sans aucune hésitation affirmer que le pari a été tenu. La preuve a été apportée que les voies à emprunter sont multiples. Des ressources de l'épigraphie et de la toponymie à celles de la littérature de fiction favorable aux morisques, des apports des sources judiciaires à ceux des sources notariales, les pages qui précèdent apportent maints exemples sur lesquels nous devons méditer. La diversité des approches révèle l'existence de voix multiples qui plus ou moins discrètes cherchent à se faire entendre. Déjà, à l'heure de l'établissement du programme, María Jesús Rubiera et Míkel de Epalza en avaient eu l'intuition. De la voix des mudejars à celle des morisques, de la voix des aragonais à celle des grenadins, de la voix de la tradition à celle de l'exil ils avaient souligné la richesse des angles d'attaque selon le statut, le lieu, le temps, le support matériel. Et si les voix directes des mudejars et morisques restent rares, les voix indirectes sont innombrables pour peu que nous voulions bien leur prêter attention. C'est là, indéniablement, l'enseignement majeur de notre rencontre.

* École des Hautes Études, París. 
La démarche qui a été la nôtre nous amène à faire le constat de l'infinie diversité des situations mudejares et morisques. Ce n'est pas une révélation mais jamais peut-être le fait n'ait apparu aussi clairement. En effet on a souvent tendance à vouloir trop simplifier et à considérer implicitement que la communauté est monolithique. Nous venons d'avoir une récente et éloquente illustration de cette tendance avec la publication du livre d'Álvaro Galmés de Fuentes Los Moriscos (desde su misma orilla) ${ }^{1}$. Le grand spécialiste de la littérature aljamiada n'hésite pas à affirmer "nunca los moriscos, como grupo social -prescindiendo naturalmente de individuos- estuvieron en trance de asimilación". Et dans sa brève conclusion (27 lignes concises), il emploie indistinctement le singulier (una comunidad morisca, esta comunidad, ese islam críptico) ou le pluriel (los moriscos) mais toujours pour envisager "la nación" comme un bloc sans nuance, sans fissure ${ }^{2}$. Si je comprends bien sa pensée, les parcours divergents à la norme identitaire n'ont pu être que rares et individuels. Pourtant on ne peut oublier que l'une des approches les plus fécondes des réalités mudejar et morisque, durant ces vingt dernières années, a été celle que l'on peut appeler régionale. Personne ne peut prétendre aujourd'hui qu'en termes d'identité ou de pratique religieuse, les morisques castillains aient été comparables aux valenciens et les grenadins aux aragonais ${ }^{3}$. Mais précisément sans remettre en cause cette pertinence nous pouvons désormais aller plus loin. Il est nécessaire de s'interroger sur la cohérence des groupes sociaux tels que celui des notables grenadins ou sur les caractéristiques de telle ou telle communauté villageoise. La connsissance approfondie de petites entités qui passe par l'exploitation prioritaire mais non exclusive des archives notariales nous permettra de mieux repérer à la fois normes de comportements et les écarts aux normes. Il faut bien comprendre une bonne fois pour toutes que mudejars et morisques ne vivent pas en vase clos.

En somme je revendique pour l'étude de ces minorités, un recours adapté à la démarche et aux techniques de la micro-histoire. S'attacher à des groupes aux effectifs limités, c'est se donner les moyens de comprendre dans le cadre de la vie quotidienne, les solidarités, les clivages et les conflits internes à la moindre cellule, les échanges et les heurts avec les vieux-chrétiens qu'ils fussent physiquement proches ou lointains. Dans cette perspective, la

1. Álvaro Galmés de Fuentes, Los Moriscos (desde su misma orilla), Madrid, 1993, p. 124.

2. Ibídem, pp. 135-136.

3. J'emploi le terme régional dans un sens ample qui s'applique à des études pouvant avoir pour objet une zone géographique réduite à une grosse ville ou à une micro-région. Je songe ainsi aux livres de Rafael Benitez SÁnchez-Blanco, Moriscos y Cristianos en el Condado de Casares, Cordoue, 1982; Juan ARANDA DONCEL, Los Moriscos en tierras de Córdoba, Cordoue, 1984; Serafín de TAPIA SÁNCHEZ, La comunidad morisca de Ávila, Salamanque, 1991; Aurelio García López, Moriscos en tierras de Uceda y Guadalajara (1502-1610), Madrid, 1992; Santiago La PARra López, Los Borja y los Moriscos, Valence, 1992. 
réalisation de monographies est hautement souhaitable à condition toutefois que les localités choisies ne le soient pas au hasard mais en fonction de problèmes précis et de l'existence de ducuments permettant de les résoudre. Le principal écueil de l'entreprise est de sombrer dans le détail non significatif. A condition, comme dirait Pierre Vilar, de penser historiquement la question mudejar et morisque, celui-ci peut être évité.

Je voudrais ici apporter quelques éléments complémentaires à l'édifice que nous avons tenté de construire. Ils appartiennent à deux domaines qu'à prime abord tout, géographiquement et socialement, sépare. Le premier ensemble concerne le milieu des colaboracionistas ou colaboradores grenadins sur lesquels peu à peu des éclairages de qualité on été portés. Le second s'attache à deux communautés villageoises de la Ribera Valencienne, Benimodo et Carlet, composées de gens modestes, sans rapport avec une quelconque élite culturelle. Les deux groupes ont cependant en commun le fait de constituer des observatoires privilégiés aux contours précis d'où se font entendre des voix s'exprimant sans le mondre filtre, des voix "directes", et sur lesquelles une analyse fine, sensible aux particularités, aux stratégies et aux enjeux peut être appliquée.

Avant de donner la parole à quelques membres de groupe colaborador ou colaboracionista, il me semble opportun de s'arrêter au vocabulaires que nous employons puis de rappeler les traits principaux du milieu. C'est à Antonio Domínguez Ortiz -ce que l'on a tendance à oublier-que l'on doit la première étude attirant l'attention sur ce phénomène $e^{4}$. Il faut relire les pages qu'il a consacrées à l'hommage à Antonio Marín Ocete. Passant en revue onze personnages ou familles il écrit précisément ceci "todos o casi todos ellos fueron lo que, en lenguaje actual llamaríamos colaboracionistas. Unos por interés, otros quizás por convicción, estuvieron al lado de los cristianos viejos durante la revuelta de 1568-1571". Vingt ans après il n'y a pas une virgule à retrancher à cette phrase. Peu après je me suis également intéressé aux mêmes individus, d'un double point de vue. J'ai tenté d'une part de montrer la continuité des choix politiques de diverses parentèles depuis la fin du XVe siècle, d'autre part de souligner la place de celles-ci dans la Grenade d'avant et après $1570^{5}$. A dessein j'ai fourni, ça et là, l'exemple de carrières qu'Antonio Domínguez Ortiz n'avait pas retracées laissant délibérément comme lui dans l'ombre l'exemple le plus passionant, celui des

4. Antonio DOMínGuez ORTIZ, «Algunos documentos sobre moriscos granadinos», in Miscelánea de Estudios dedicados al profesor Antonio Marín Ocete, Grenade, 1974, vol. I, pp. 247-254.

5. Voir Bernard VINCENT, «Los moriscos que permanecieron en el Reino de Granada después de la expulsión de 1570», in Andalucía en la edad moderna: economía y sociedad, Grenade, 1985, pp. 267-286, et «La familia morisca» in Minorías y marginados en la España del siglo XVI, Grenade, 1987, pp. 7-29. Ces deux apports furent une première fois publiées respectivement en 1981 et 1974. 
Granada-Venegas qui mérite un ouvrage à lui seul. Ces dernières années ont proliféré les travaux apportant à la fois des compléments quant à l'information et des approfondissements quant à la reflexion. Tandis qu'Ángel Galán s'attachait au colaboracionismo des mudejars grenadins, distinguant trois degrés (oligarchie, pouvoirs intermédiaires et une troisième composante aux contours plus flous $)^{6}$. Enrique Soria montrait à quel point, à l'initiative de la Couronne, l'intégration de l'aristocratie nasride ou de ce qu'il en restait avait été poussée dans la Grenade du XVIe siècle ${ }^{7}$. Leurs apports étaient nourris aussi des recherches de Manuel Espinar et Juan Grima sur Cidi Yahya (Pedro de Granada), d'Antonio Muñoz Buendía sur la zone d'Almería et de Javier Castillo sur celle de Baza ${ }^{8}$. Tout récemment Valeriano Sánchez Ramos est revenu sur le sujet en commentant l'attitude des colaboracionistas lors du soulèvement morisque de 1568-1570. Il distingue essentiellement les nouveauxchrétiens participant activement aux opérations dans le camp royal à ceux qui recherchent à sceller la paix entre les deux adversaires. Ces derniers appartiendraient à l'oligarchie alors que les premiers se recruteraient surtout parmi les détenteurs du pouvoir en milieu rural.

Me resérvant la possibilité de débattre ailleurs divers aspects de ce dossier désormais extrêmement riche, je me contenterai, pour le moment, de quelques remarques qui me semblent utiles pour notre colloque. Depuis le travail pionnier d'Antonio Domínguez Ortiz on avait privilégié le terme colaboracionista même si personellement $j$ 'avais retenu celui de colaborador sans suffisamment m'en expliquer. Valeriano Sánchez Ramos propose d'établir la distinction entre les deux mots ${ }^{9}$. Je crois qu'il a raison. Le colaboracionista est celui qui se range du côté du pouvoir par calcul politique ou esprit de lucre,

6. Ángel GalÁN SÁNCHEZ, «Poder cristiano y 'colaboracionismo' mudéjar» in Estudios sobre Málaga y el Reino de Granada en el $V$ Centenario de la Conquista, ed. José Enrique López DE CocA, Málaga, 1987, pp. 271-289. Notons que l'auteur de cette étude, par ailleurs très riche, fait une affirmation étrange "Sin embargo, está sin estudiar el efecto que en la sociedad morisca tuvieron los descendientes de estos primeros colaboracionistas y de otros que se encaminaron en el camino hasta 1572 " et une autre qui me paraît devoir être très nuancée "es indudable que la Corona fracasó en sus pretensiones de integrar a los moriscos en la sociedad cristiano-vieja" (p. 289).

7. Enrique SORIA MESA, «De la conquista a la asimilación. La integración de la aristocracia nazarí en la oligarquía granadina. Siglos XV-XVII», Áreas, 14, 1992, pp. 49-64.

8. Manuel Espinar MOREno et Juan Grima Cervantes, «Un personaje almeriense en las crónicas musulmanas y cristianas. El infante Cidi Yahya Alnayar (1435?-1506): su papel en la guerra de Granada», Boletín del Instituto de Estudios Almerienses, VII, 1987, pp. 57-83; M. Espinar Moreno et J. Grima Cervantes, "Testamento y muerte de don Pedro de Granada», Mayurqa a, 22, 1989, pp. 239-254; Antonio MUÑOz BUENDíA, «Supervivencia de la población morisca en Almería después de la expulsión de 1570: ejemplo de algunas familias», Hespérides. Actas del IX Congreso de profesores-investigadores, El Ejido (Almería), 1990, pp. 503537; Javier CASTILLO FERNÁNDEZ, «El origen del concejo y la formación de la oligarquía ciudadana en Baza (1492-1520)», Chronica Nova, XX, 1992, pp. 39-73.

9. Valeriano SÁNCHEZ RAMOS, «Los Moriscos que ganaron la guerra», Mélanges Louis Cardaillac, Zaghouan, 1995, tome II, pp. 613-627. 
par intérêt disait déjà Antonio Domínguez Ortiz; le colaborador est celui qui, convaincu de l'impossibilité d'emprunter un autre chemin, participe aux affaires mais dans le but de préserver ce qui peut l'être de l'identité morisque. Le Dictionnaire de la Real Academia de la Lengua souligne bien la connotation positive ou neutre de colaborador et celle péjorative de colaboracionista.

Le groupe des colaboracionistas au sens strict du terme "el que presta su colaboración a un régimen político que la mayoría de los ciudadanos considera antipatriótico" existe dans l'Espagne morisque comme partout ailleurs. Mais c'est bien le groupe des colaboradores autrement plus original qui doit retenir notre attention. II est celui qui, au sein de la communauté morisque représente la politique la plus élaborée, la plus consciente. Constitué pour l'essentiel -encore faudrait-il y regarder de plus près- par des membres de l'oligarchie, il a plus que tout autre les moyens de s'exprimer. Ce monde d'une double culture, qui tente désespérément de peser sur le cours d'évenements qu'il devine inéluctables ("Trataba de hacer oír su voz en un medio hostil" rappela naguère Francisco Márquez Villanueva), ne peut que passionner les historiens du socioculturel.

Précisement plusieurs membres du groupe ont fait l'objet d'etudes fouillées. Je songe au travail du père Cabanelas sur Alonso del Castillo et à celui de Francisco Márquez Villanueva sur Miguel de Luna ${ }^{10}$. Mais il importe désomais de tenter de montrer l'existence d'un véritable courant qui n'a cessé de s'exprimer au XVIe siècle et même au-delà. Deux témoignages supplémentaires me semblent à cet égard pouvoir être invoqués. Le premier qui n'étonnera personne est le fameux mémoire de Francisco Núñez Muley. Fameux ct aussi inconnu car combien de personnes l'ont lu en intégralité? ${ }^{11}$.

Le mémoire de 1567 est le dernier jalon d'un parcours entièrement consacré à la défense de la communauté morisque du royaume de Grenade. Probablement né vers 1490, descendant des Mérinides, Francisco Núñez Muley a accompli une carrière d'une loyauté sans faille à l'égard des souverains espagnols successifs, Isabelle et Ferdinand, Charles Quint, Philippe II. Il dit avoir été page de l'archevêque de Grenade, Hernando de Talavera qu'il aurait accompagné au cours d'une visite des Alpujarras en 1502. Lorsque Charles de Gand fit son premier voyage en Espagne en 1517, Francisco Núñez Muley accompagna le marquis de Mondéjar, capitaine général du ro-

10. Francisco Márquez Villanueva, El problema morisco (desde otras laderas), Madrid, 1991, pp. 45-97. Ce texte avait été publié antérieurement dans la Nueva Revista de Filología Hispánica, XXX, 1981, pp 359-395.

11. Ce texte a fait l'objet de deux publications in extenso d'abord par Raymond FOULCHEDelbosc, «Memorial de Francisco Núñez Muley», Revue Hispanique, 1899, pp. 205-239; Kenneth GARRAD, "The Original Memorial of Don Francisco Núñez Muley», Atlante, II, $\mathrm{n}^{\circ}$ 4, 1954, pp. 168-226: la version de Foulché-Delbosc va être reprise dans la réédition du livre de Antonio Gallego Burín et Alfonso Gamir Sandoval, Los Moriscos del Reino de Granada según el sinodo de Guadix en 1554, Granada, 1968, à paraître, Grenade, 1996. 
yaume de Grenade, pour rendre hommage, à Valladolid, au nouveau roi. Il semble avoir été percepteur de l'impôt de farda dont les morisques devaient s'acquitter et son fils Alonso Martínez de Santiago lui a succédé dans cette charge. Le père et le fils resultent privilèges et gratifications. Francisco Núnez Muley fut le 26 octobre 1526 autorisé à porter les armes. Comme huit autres notables morisques il jouissait d'un bénéfice lié à l'Alhambra lui assurant 9000 maravedis par an. Celui-ci fut transféré à son fils également le 26 octobre 1526. Le 3 novembre de la même année Francisco obtint un don royal de 20 ducats. Alonso Martínez de Santiago reçut une grâce similaire en $1543^{12}$. Le courtisan, l'agent royal, était admirablement placé pour faire entendre sa voix. Il ne s'en est pas privé. Déjà en 1513, alors qu'il devait ne pas avoir plus d'une vingtaine d'années, il faisait partie d'une délégation tentant de s'opposer à l'application de mesures restreignant ou éliminant des pratiques vestimentaires ou alimentaires. En 1523, il est avéré qu'il s'inquiétait auprès des autorités des dispositions prises à propos du parrainage. Luimême fait allusion à sa présence dans une ambassade reçue par l'archevêque Gaspar de Ávalos -donc obligatoirement entre 1529 et 1541- et désireuse de justifier le port du voile par les femmes. Et il indique avoir très souvent conversé avec les inquisiteurs. Fidèle à lui-même, l'infatigable avocat ne pouvait pas ne pas être ému par le programme acculturateur mis en place par la junte de Madrid de 1566.

L'argumentation de Francisco Núñez Muley est pour l'essentiel connue. Les morisques que lui appelle de manière significative naturales (ce terme figure plus de 60 fois dans le texte) ont des pratiques ancestrales qui n'ont rien à voir avec celles du Maroc ou de Turquie et dont la singularité est comparable à celle des chrétiens de Jérusalem ou de Malte. Elles sont un patrimoine intouchable. De surcroît la suppression de la plupart d'entre elles serait source de difficultés économiques qui affecterait la Couronne; ainsi l'industrie de la soie serait menacée. La démarche est indéniablement ingénieuse et habile. Mais la rhétorique même n'est pas moins intéressante. Núñez Muley se presente comme un patriarche dont la sagesse mérite respect et attention. Il est la mémoire de la communauté parce qu'il est âgé et parce qu'il a été un acteur privilégié de la convivance. 14 fois il emploie le terme memoria ou memorias (hay memoria, memoria antigua, estas memorias, amparar las memorias ...). Et 7 fois il conjugue le verbe acordarse, ainsi lorsqu'il fait référence aux offices que présidait l'archevêque Hernando de Talavera. Le prélat, rappelle-t-il, disait à l'adresse des morisques "y bara ficum et non dominus vobiscum" et de commenter "esto me acuerda dello como si fuese ayer, en el ano de quinientos y dos". Inlassablement Núñez Muley nourrit sa plaidoirie de faits, de details qui sont autant de pièces à conviction. Tout juste avoue-t-il par trois fois de petites défaillances "no me acuerdo", commet-il une petite erreur de date

12. Archives Générales de Simancas, Camara Cedulas 255, fol. 366 el 382 v . 
concernant la mort de Ferdinand le Catholique (1517 selon lui), a-t-il une hésitation sur celle d'une entrevue avec l'archevêque Gaspar de Ávalos (1532 ou 1540 mais les deux options sont possibles!). Partout ailleurs il multiplie les réferences précises, par exemple à des évènements éloignés dans le temps, aux capitulations de 1491 qui, assure-t-il, comprennent une quarantaine d'articles (elles en ont 47!), au mouvement des Comuneros et à la reunion de la Capilla Real de Grenade en 1526. Ou aux dates des cédules royales visant des coutumes des morisques en 1508, 1511, 1512, 1513, etc..., toutes rigoureusement exactes. Il arrive même à se souvenir qui était corregidor au moment de la promulgation de tel ou tel texte. Tout est gravé dans sa mémoire. Ayant ainsi accumulé les preuves, l'avocat morisque peut prétendre être détenteur de vérités qui ont été occultées et qu'il est nécessaire de rappeler. En un mot de la vérité, terme qu'il emploie d'ailleurs a 9 reprises. Sans oublier les neuf variations sur es notorio, siendo notorio, notoriamente... Francisco Núñez Muley est la conscience du royaume de Grenade. II dit haut et fort le vrai.

L'ecrit $d u$ notable grenadin est d'autant plus précieux qu'il s'avance sans aucun masque. Il n'y a pas de voix plus directe que la sienne. Ecoutonsle. II n'emploie pas moins de 21 fois l'adjectif santo, l'accolant de préference -15 fois- à fe. Mais il est aussi question trois fois du santo arzobispo Hernando de Talavera -ce vibrant hommage mérite d'être souligné-, des santos sacramentos, de la santa casa de Jérusalem et même du santo Oficio de la Inquisición, chacun une fois. Le nouveau chrétien est dans son vocabulaire au-dessus de tout soupçon. Ne va-t-il pas jusqu'au dire "para el que hiziere lo que no deve contra la santa fe catolica, para esto son los señores inquisidores para lo castigar como lo castigan". L'action du terrible tribunal ne suscite donc chez lui aucune réserve. De surcroît lorsqu'il désigne les musulmans, il n'a qu'un seul mot, 14 fois utilisé, celui de seta. Les adversaires les plus déclarés des morisques ne diraient pas autre chose. Et par deux fois, Francisco Núñez Muley se réfère a la sagrada escritura pour justifier l'existence des bains publics. II commente le chapitre X1, versets 2 à 4 du livre de Samuel où est narrée la faute de David "... No se pueda negar en que dize que si Betsabe no se lavara, David no pecara y hera profeta...". Enfin les dernières lignes du post-scriptum du mémoire sont un rappel éloquent des deux premiers commandements, tel que les énoncent Mathieu et Marc "tu aimeras le seigneur ton Dieu de tout ton coeur, de toute ton âme et de tout ton esprit" et "tu aimeras ton prochain comme toi-même".

Cet homme nourri des écritures saintes est aussi d'une loyauté totale à l'égard des princes. Il est question chemin faisant de la reine doña Juana nuestra señora et de l'emperador rey nuestro señor. Pourtant tout en appartenant sans la moindre hésitation au monde des chrétiens (dios nuestro señor dit-il encore deux fois) et en étant un sujet modèle, il ne renonce nullement à son appartenance a la communauté morisque. Les 11 adjectifs possessifs disséminés dans 
le texte sont lourds de signification. Si cinq, comme, nous venons de le voir, se rapportent à Dieu, à la foi ou aux souverains, six relèvent d'un autre registre, mis ancianos, nuestros viejos y mas ancianos, naestra nacion ( 3 fois), mi sangre, ultime cri que le vieil homme profère. Francisco Núñez Muley assume un double héritage et n'entend nullement renoncer à l'un des deux. Avant de signer son grimoire il rédige deux lignes qui résument admirablement sa position "porque mi intencion ha sido y es muy buena en servir a dios nuesto senor y a su majestad y a los naturales sus vasallos deste reino, pues son mi sangre y soy obligado a ello e no los puedo negar". II revendique ainsi le rôle de passeur culturel qu'il a exercé tout au long de son existence.

Non sans courage, il rappelle dès le début ce que fut la conversion au christianisme, obtenue par la force et à l'encontre de la lettre et de l'esprit des Capitulations signées par les Rois Catholiques et Boadbil. Et il décrit la dégradation des conditions faites aux morisques "antens cada dia peor y mas maltratados en todo y por todas vias y modos ...". Il prône implicitement un retour à la politique, au climat du temps du saint archevêque Hernando de Talavera à qui il voue une vénération sans bornes. Mais soixante ans ont passé et la communauté morisque est menacée de perdre son identité. Le vieillard en est désespéré et dans ce dernier combat il engage toutes ses forces. On sent que peu à peu il est débordé par l'émotion et qu'il finit par ne plus contrôler sa plume. Alors qu'il a privilégié longtemps pour désigner les minoritaires le terme naturales, il laisse échapper en tout six fois le mot morisques dont quatre fois dans les tout derniers paragraphes du document.

On ne sait rien des derniers moments de la vie de Francisco Núñez Muley. Probablement s'était-il déjà éteint quand le soulèvement des morisques embrassa tout le royaume de Grenade à la fin de l'année 1568. Mais il y a trace d'autres membres de sa parentèle en particulier d'Hernando Muley qui affirme être son sobrino segundo et d'Andres Muley. L'un et l'autre furent expulsés de Grenade. Probablement Hernando put-il revenir avant 1580 , date a laquelle un Fernando Muley figure dans la liste des morisques autorisés à demeurer sur place. Est-ce lui qui accompagne au printemps 1583 le traducteur Alonso del Castillo dans son déplacement de Grenade à Madrid? ${ }^{13}$. Notons toutefois que nous retrouvons un Hernando Muley et un Andrés Muley a la tête du complot découvert à Séville en $1580^{14}$. Même si, au stade actuel de la recherche la confusion est grande entre les divers membres de la famille, il semble acquis que par des voies diverses, plusieurs d'entre eux ont participé à des entreprises visant à empêcher la disparition de l'identité morisque.

13. Darío Cabanelas Rodríguez, El morisco granadino Alonso del Castillo, Grenade, 1965, p. 160.

14. Antonio DOMíNGUEZ ORTIZ, «Desventuras de dos moriscos granadinos», Homenaje a Jacinto Bosch Vilá, Grenade, 1991. 
Je viens de faire une allusion à Alonso del Castillo, l'un des principaux artisans du montage de l'affaire des livres du Sacromonte ${ }^{15}$. On sait que le 18 avril 1588 fut découverte au pied de la Torre Turpiana, le minaret de la grande mosquée de Grenade, une caisse de plomb contenant des reliques et un parchemin indiquant que celles-ci avaient été confiées par l'archevêque San Cecilio à un disciple. Et le 21 février 1595 apparaissait sur le Sacro Monte un livre de plomb. Dix autres allaient être découverts. Ainsi commençait une ambitieuse tentative de syncrétisme etnico-religieuse dont les principaux auteurs étaient deux morisques, Alonso del Castillo précisément et aussi Miguel de Luna, le premier étant peut-être le beau-père du second ${ }^{16}$.

Miguel de Luna eut de Doña María plusieurs enfants dont un Juanico fait l'objet d'une lettre adressée à l'archevêque de Grenade, Pedro de Castro en janvier 1610. Luna souhaite que son fils soit ordonné ${ }^{17}$. Mais on trouve aussi dans les dossiers du tribunal de l'Inquisition de Grenade la relation de la cause d'une certain Alonso de Luna, 50 ans, condamné en 1619 à la prison perpétuelle pour avoir proféré des propositions hérétiques ${ }^{18}$. Il est infiniment regrettable de ne pas disposer du procès de l'intéressé afin de mieux s'approcher de sa véritable voix. Pourtant la longueur et la précision de la relation de cause permettent de formuler des hypothèses. Alonso de Luna serait né à Linares et aurait séjourné en France oú il aurait fréquenté des musulmans. A Grenade, à l'âge de 18 à 20 an, donc vers 1585-1590, il aurait embrassé l'Islam. Parcours étrange et suspect. Médecin, il connaîtrait les langues castillane, latine, italienne et arabe. Les témoins le disent morisque et en prison il fait ses ablutions. Lui-même reconnaît avoir récité les cinq prières quotidiennes du croyant musulman, avoir observé le jeûne du Ramadan et connaître le Coran par coeur. Il a adressé une lettre au pape et trois autres au roi d'Espagne, écrites partiellement en arabe, où il annonce le temps proche de la Résurrection. Transporté une nuit au sixième ciel par les anges, il a vu Dieu qui lui a annoncé qu'il serait l'agent de la conversion générale au catholicisme. Mais la langue de la conversion serait l'arabe. Dieu lui a révélé que toute la vérité évangélique se trouve dans les livres du mont sacré de Grenade.

Les inquisiteurs sont perplexes et en marge se trouve la mention "les inquisiteurs cherchent à savoir si l'accusé ne fut pas interprète des livres du mont sacré de Grenade". Il n'y a pas de fumée sans feu. Alonso de Luna dit encore que son père, le docteur Luna, contrairement aux apparences n'est pas mort.

15. Miguel José Hagerty, Los libros plúmbeos del Sacromonte, Madrid, 1980; Julio Caro Baroja, Las falsificaciones de la Historia (en relación con la de España), Barcelone, 1992, pp. 115-158.

16. D. Cabanelas Rodriguez, op. cit., p. 231

17. D. Cabanelas Rodríguez, "Cartas del morisco granadino Miguel de Luna», Miscelánea de Estudios Árabes y Hebraicos, 1965, p. 43.

18. Archives Historiques Nationales de Madrid, Section Inquisition, legajo 1953. 
Même s'il est difficile de démêler les fils de l'écheveau, je suis enclin à croire qu'Alonso de Luna est bien un fils de Miguel, décédé en 1615 et qu'il porte le prénom de son grand-père maternel Alonso del Castillo. Sa déposition qui par deux fois fait explicitement référence aux livres de plomb a bien des points communes avec leur contenu, en particulier quant à la place de la langue arabe. Le messianisme d'Alonso de Luna serait dans ces conditions une nouvelle version des efforts desespérés qu'un groupe de morisques cultivés, de Francisco Núñez Muley à Alonso de Luna, aurait inlassablement consentis, au moins des années 1560 aux années 1620 , pour sauver une partie de son héritage culturel. Bien entendu il importe de reconstituer le réseau mais aussi de retrouver tous les messages que celui-ci a pu diffuser. Francisco Núñez Muley et Alonso de Luna appartenaient bien l'un et l'autre à ce que Rolena Adorno a, à la suite d'Angel Rama, appelé "la ciudad letrada"19.

Avec les paysans de Benimodo et de Carlet nous abordons un aspect radicalement différent de la prise de parole morisque. Il s'agit de voix indirectes, contrairement au cas de Francisco Núñez Muley, puisque nous ne pouvons les entendre qu'à travers le prisme de l'inquisition. Elles n'en sont ni moins fortes ni moins éloquentes ce qui me permet de souligner au passage l'intérêt que représentent les sources inquisitoriales pour l'étude des minorités. Il est curieux de constater que dans leurs ouvrages récents, aux points de vue radicalement divergents, Francisco Márquez Villanueva et Álvaro Galmés de Fuentes se rejoignent cependant dans leur condamnation du recours à ce type de documents. Le premier parle de sources "empoisonnées", le second de "vision partisane et sectaire" qui en dérive dans les études ${ }^{20}$. Or l'historien sait bien que toutes les sources sont empoisonnées et que derrière chacune se trouve une personnalité, un tempérament, une idéologie, une stratégie. Il ne faudrait pas qu'une censure hâtive conduise au rejet systématique de données foisonnantes que le nécessaire examen critique peut rendre très précieuses.

Je vais tenter d'en apporter une illustration immédiate, utile aux débats de ce colloque. En 1574, plusieurs villages de la Ribera valencienne, Benimuslem. El Pujol, Alcira, Benimodo, Carlet ont fait l'objet d'une visite inquisitoriale au cours de laquelle un édit de grâce concernant les morisques fut promulgué. Pour en bénéficier les habitants de ces lieux devaient se présenter devant $l^{\prime}$ inquisiteur et confesser gestes et pratiques interdits ${ }^{21}$. $\mathrm{Au}$

19. Rolena Adorno, Cronista y principe. La obra de Guaman Poma de Ayala, Lima, 1989, pp. $225-$ 245. L'auteur y fait une suggestive confrontation entre les textes de Francisco Núñez Muley et Guaman Poma de Ayala.

20. Francisco MÁrqueZ VIllanUeVa emploie le terme "enturbiadas", op. cit., p. 3; voir surtout p. 166 et sv. Álvaro GALMÉs DE FüNTES, op. cit., p. 11.

21. A.H.N., Madrid, section Inquisition, legajos 544 et $803 / 20$ et archives privées. Voir une présentation du dossier dans B. VINCENT, "Le chat et les souris, inquisiteur et morisques à Benimodo (1574)», Estudis, XX, 1994, pp. 277-289. 
total 910 crypto-musulmans au moins ont été auditionnés et pour 561 d'entre eux les dépositions ont été retrouvées. Les deux ensemble les plus importants sont ceux de Benimodo (159 confessions) et de Carlet (317 confessions). Le document-type a l'aspect $d^{\prime}$ 'une fiche policière où ont été consignées les réponses fournies par les intéressés. Ceux-ci, parfaitement conscients des enjeux, cherchent à ne pas tomber dans les pièges des interrogateurs et à ne faire aucune révélation susceptible de nuire à un autre membre de la communauté. La ligne de défense générale est claire: il faut préserver les leaders et les initiateurs.

Ce document passionnant, qui permet de connaître à la fois des pratiques religieuses et culturelles et le réseau des alliances matrimoniales, ne nous fait d'ordinaire pas entendre les voix des morisques. A la médiation du greffier s'ajoute l'insécurité des interrogés. Cependant une lecture attentive et la confrontation de plusieurs dépositions entre elles mettent en lumière silences, oublis conscients, mensonges toujours lourds de signification. La défense passive ayant ses limites, le chercheur, comme l'inquisiteur, fait une ample moisson d'informations cet se convainct des écarts existant d'un village à un autre et de l'ampleur de la résistance au plan général.

II y a plus. Sur ce fond d'attitudes communes se détache un petit groupe, composé de 34 personnes ( 20 de Carlet et 14 de Benimodo) qui n'accepte pas d'entrer dans le jeu des inquisiteurs. Au lieu d'admettre la réalité des faits la plupart de ces derniers -les 20 de Carlet et 4 de Benimodo- préfèrent nier en bloc et prétendre être de parfaits chrétiens. Pour les besoins de la cause, $l^{\prime}$ interrogateur cherche à pousser les morisques dans leurs retranchements et avec chacun s'instaure un dialogue que le scribe retranscrit au moins partiellement. Nous avons alors l'impression d'assister aux échanges et d'entendre directement la voix de modestes paysans morisques.

La posture adoptée n'allait pas sans danger car la réplique de l'inquisiteur était imparable. Elle était composée de deux volets qui apparaissent bien par exemple à travers le cas de Geyma Badadel, 16 ans, habitante de Carlet qui dépose le 30 juin. Lorsque celle-ci proclame l'orthodoxie de sa foi, il lui est immédiatement rétorqué qu'être fille de musulmans (le texte dit moro et non morisco ou cristiano nuevo), avoir été élevée en milieu musulman, porter un prénom musulman, utiliser des vêtements à la musulmane sont autant d'indices qui la rendent non crédible. Elle doit donc passer aux aveux. Geyma persiste et affirme qu'elle a toujours eu l'intention d'être chrétienne. Pedro de Zárate, l'inquisiteur lui demande alors de réciter les prières chrétiennes qu'elle connaît. C'est l'impasse: Geyma qui ne sait rien s'empresse de dire qu'elle va les apprendre. L'entretien se termine par une solennelle et menaçante admonestation. La jeune morisque plongée dans la perplexité et l'angoisse finit par comparaître une deuxième fois le 25 août. Elle déclare avoir initialement menti parce qu'elle avait peur et elle prononce les mots qu'attendait son interlocuteur. 
Le dernier scénario, le plus rare et le plus dramatique, concerne dix habitants de Benimodo. Eux refusent longtemps d'exprimer le regret d'avoir accompli des gestes prohibés et surtout d'avoir professé l'islam. Si deux de ces dix irréductibles rendent les armes au bout de l'insoutenable interrogatoire, huit resistent à toutes les pressions et tiennent la dragée haute à l'inquisiteur. Ce n'est qu'un mois plus tard et selon toute vraisemblance sur le conseil de leurs proches qu'ils rompent avec une attitude à hauts risques et consentent à proférer les paroles d'adhésion à la foi chrétienne qui leur font tant horreur. On trouvera en annexe la transcription de l'un de ces dialogues car celui-ci rend compte mieux que tout commentaire de l'exceptionnelle tension à laquelle étaient soumis les déclarants.

L'éloquence de la voix des plus réductibles est impressionnante. D'abord il est clair qu'eux et eux seuls parlent vrai. Pedro de Zárate ne peut être dupe des biais empruntés par l'immense majorité de ses interlocuteurs. Les rares individus qui ne plient pas parlent au nom de la communauté et rendent dérisoires et caducs les aveux de tous les autres. Ensuite la composition du groupe ne laisse aucun espoir à l'inquisition. S'il s'était agi des morisques les plus âgés de Benimodo et de Carlet, Zárate aurait pu penser que la conversion serait bientôt acquise. Mais parmi les 34 inflexibles, 22 ont moins de 25 ans. Et constatons que parmi les 34, 29 sont des femmes. Une fois de plus est mis en lumière leur rôle essentiel dans la lutte pour le maintien de la foi musulmane. Xumeyca Xat à qui l'on demande si elle se repent d'avoir été musulmane dit simplement non.

Xumeyca Xat qui a 50 ans, Nexma Zolaytan 14 ans ou Fatima Ratal 14 ans et quelques autres sont les porte-parole de la communauté. A quelques années de distance elles sont l'égal de Francisco Núñez Muley. Elles n'ont ni sa rhétorique ni sa formation, ni sa stratégie ni ses perspectives mais elles sont tout autant convaincantes et émouvantes. Elles nous administrent aussi la preuve que l'expression morisque n'est pas le monopole des lettrés. Au contraire, ô ironie, nous pouvons la débusquer jusqu'au coeur des sources empoisonnées, incapables de les celer.

\section{APPENDICE DOCUMENTAIRE}

\section{1}

A.H.N. Inquisición

leg. 1953

Alonso de LUNA preso y penitenciado en la Inquisición de Murcia.

"por testificacion que uvo contra el de aver dicho que un hombre que fue condenado y relaxado por el santo officio avia muerto de puro honrado y que no estava determinado por los theologos si un hombre estava obligado a confesar su delito aunque estuviese testificado dello y que bastava para que uno se salvase confesarse a dios en su corazon y 
que murio christiano y de aver dicho tambien que avia mejor generacion que la de los moros porque nuestra señora la virgen Maria le avia aprovado por la mejor de todas y de que tratando de ciertas personas presas en la Inquisicion dixo que saldrian castigadas pero sin culpa y que los mas que ally se castigaran era sin tenerla por testigos falsos - y la penitencia fue que en la sala del Audiencia se le leyese su sentencia. Abjurase de levi y fuese reprehendido y advedido gravemente y desterrado del districto desta y de aquella Inquisicion por seis anos y si los quebrantase los cumpliese en las galeras al remo y sin sueldo y pagase docientos ducados y aviendose executado se le dieron clertos terminos para salir a cumplir el destierro y en este intermedio fue testificado en este santo oficio de aver dicho las posiciones siguientes:

$1^{\circ}$ Visitando a cierta persona enferma dixo que no se avia de morir de aquella enfermedad porque era inmortal y que no aviamos nacido para morir ni queria Dios que muriesemos y que los que morian era por no azertarles los medicos las curas contestaron en esta proposicion cuatro testigos ratificados, calificose por heretica.

$2^{\circ}$ Dixo en el mismo proposito y platica que aunque uno muriese en pecado mortal no se iria al infierno porque era dios misericordioso y lo llevaria al cielo. Contestaron tres testigos ratificados y uno dellos añadio que diciendole que despues de la muerte avia de aver infierno y gloria para siempre. Respondio que no era el infierno para siempre ni Dios condenava a nadie por tanto tiempo. Calificose por heretica contra articutos de fe. $Y$ tres de los dichos testigos depusieron que el dicho reo era morisco y de casta de ellos. Votose la sumaria a reclusion en las secretas que se hiciese su causa con el y se recluyo en 22/VI/1618 y en 25 del mismo.

\section{Audiencias}

Dixo ser natural de la villa de Linares de hedad de 50 anos y que avra estudiado lalatinidad philosophia y medicina y era graduado de Iyzenciado en ella y sabia leer y escrivir en quatro lenguas castellana latina ytaliana y araviga y se tenia por christiano viejo Estuvo negativo en eI discurso de su causa pusosole la primera acusacion en 7/VII y a 30 del mismo se le dio la primera publicacion y en $8 / \mathrm{VIII}$ alego y articulo ciertas defensas que se comenzaron a hazer luego pretendiendo probar que avia tenido y tenia enfermedad de perlesia y gota coral que le avia dexado falto de memoria y de juicio y que hacia y que decia disparates.

Estando la causa en este estado nos mando nuestra senoria remitir unas cartas escriptas y firmadas de mano del Reo. La una para su sanctidad y las tres para su Majestad en que avia algunas partes escriptas en Aravigo con una calificacion que vino hecha y remitida con ellas de que estaban llenas de disparates y heregias con lenguaje al modo del que usan los moros. Para que vistas hiciesemos en la causa justicia, recivioronse en 13 del dicho mes de agosto con carta del 7 del mismo. Reconociolas y fue declarando lo que contenian que lo màs sustancial fue que estando una noche en el campo fue llevado por los Angeles o por la potencia de Dios al cuarto cielo y desde alli al sesto y que vio muchas visiones y a Dios nuestro senor sentado en su trono con sus Angeles los cuales mobian los cielos y que le dixo hijo no temes que yo te daré saviduria infusa, escribe al Rey y al Papa y diles come ya es tiempo de la Resurrection y de acavarse todas las heregias y convertírse todo el mundo a la santa fe catholica y que en los tiempos finales la vendria a socorrer la nacion Arabe y en su lengua arabiga avia de ser la conversion porque esta lengua era perfectissima y la avia elegido Dios por la mejor y con ella le alavavan los Angeles y que avia de castigar a los españoles porque no la querian admitir tiniendo como tenian todos obligacion de saberla y que no avia de quedar rincon en este mundo que no se perfecionase allanandose las Montañas y ensanchandose y convirtiondose las aguas ynutiles en Aire y a los hombres a quien Dios fuese servido les avia de dar el Paradiso terrenal que es este mundo para siempre como a criaturas menos perfectas que los Angeles y el cielo a los Angeles como mas perfectos y que el libro del Alcoran era divinarum rerum colectio y que en cumplimiento de lo 
que Dios le mando escribir las dichas cartas para que supiese su Majestad que en su tiempo avia de ser esta conversion general por medio del Reo, diciendo que tambien dios le abia revelado que en los libros del monte sancto desta ciudad estaba escripta toda la verdad catholica y evangelica y avia un libro ilegible el cual hasta ahora no se avia podido leer ni entender y le tenia Dios guardado a el para que le leyese y yuterpretase y publicase la conversion y juicio final en la forma referida y declaro tambien que lo escripto en Arabigo en las dichas cartas eran dos oraciones que la una comenzaba las alabanzas sean dadas a dios y la otra otra la de el andululey - enpero nego averlas escripto con intencion de guardar ni enseñar la secta de Mahoma y mezclo en sus confesiones algunos disparates como tambien lo estaban en las dichas cartas y entre ellos que no avia demonios y que si los avia los avia de consumir la Potencia de dios y que el doctor Luna su padre aunque era difunto no era muerto sino que le avia suvido Dios a una fabrica que esta sobre nosotros que llama Dios el Ternete atenendo porque tiene ally a los justos y buenos = todo lo cual se torno a calificar por proposiciones hereticas mahometanas dirigidas a la ensenanza, creencia y observanza de la secta de Mahoma y contenidas en el libro del Alcoran.

Demas de lo cual le testificaron dos testigos ratificados companeros de carzel de que de noche y a escusas dellos se lavava las partes del cuerpo y hazia cruzes de palillos y las pisaba y se sentaba sobre ellas y que rezando los testigos el credo y llegando a aquellas palabras deszendio a los infiernos y saco las Anymas de los sanctos Padres Dixo veis ay como no tiene Dios para siempre las Animas en el infierno que no es tan ingrato que las deje de sacar del y que avian echado de ver que los disparates que alguna vez decia y hacia eran fingidos con artificio y malicia y deste vItimo parezer fueron el Alcayde y su ayudante dos medicos y un zirujano deste santo oficio que en diversas vezes le vieron y visitaron por nuestra orden para verificacion dello.

secrestaronsele sus bienes y se le puso segunda acusacion de todo lo dicho sobrevenido en 22/XI/1618 y confeso lo mismo negando la intencion y diciendo que no estaba loco ni lo fingia sino que avian sido revelaciones de Dios, si bien padecia las dichas enfermedades $=\mathrm{y}$ fueron se haciendo la diligencias en eI resto del año ... hasta que concluya su causa para definitiva se voto en 24/IV/1619 a que fuese puesto a question de tormento sobre la intencion y diminuciones y fiction de la locura, y por estar enfermo no se pudiere (eje)qutar hasta 10/VI/1619 y antes de llegar a desnudarse confeso que desde que nacio habia aprendido el lenguaje arávigo y le avia usado donde quiere que avia estado especialmente con los Moros que ay en Francia y que abria 18 o 20 anos que aprendio en esta ciudad la dicha secta y la avia guardado haciendo el guadoc y zala y rezado las zoras del Andu y colalaguaat y ayunado en ayuno del Ramadan y zelebrado la Pascua de los Moros y que sabia todo el Alcoran y era hombre docto en la ciencia del y que del mismo Alcoran avia sacado las proposicionos y escriptos arabigos que contenian las dichas cartas y las avia escripto a fin de que se supiese la secta de Mahoma y se convirtiesen todos a ella y que para que le diesen mas credito avia dicho que eran revelaciones de Dios y que estaban en los libros del Monte sancto y que si avia mezclado disparates y fingido alguna locura Avia sido con intento de evadirse de la pena y castigo y que hasta entonces habia perseverado en la observacion de la dicha secta con intencion y pertinacia y bien se a comprobado aver sido fingimiento los disparates que decia y acia porque desde que hizo esta confesion no ha hecho ni dicho ninguno.

tornado a ver el proceso en definitiva se voto a 15/VI que en auto publico si se esperaba de proximo o sino en una iglesia fuese admitido a reconciliacion en forma con confiscacion de bienes habito y carcel perpetua y no se condeno a galeras por no ser el sujeto en ninguna manera para ello por sus enfermedades y pocas fuerzas auto particular Santiago domingo 22/XII/1619 y se entrego a la carcel de la penitencia desta inquisicion al alcayde della

(en el margen informen los inqvisidores si este reo fue interprete de los libros del monte santo de Granada). 
A.H.N. Sección Inquisición

legajo $803 / 20 \mathrm{n}^{\circ} .11$

En Carlete a veynte y un dias del dicho mes y año antel señor inquisidor doctor Pedro de Carate parescio Esperança Ratal y de mora Fatima muger de Bartolome Gazi cristiana nueva de mora vezina de Benimodol de la qual fue rescebido juramento en forma y prometio de dezir verdad y declaro ser de edad de quatorze anos

preguntada dixo que viene a confesar anta su senoria lo que haste aora a hecho de mora par gozar de la gracia y perdon que tiene publicada a los cristianos nuevos de Benimodol

preguntada dixo que lo que a hecho siendo cristiana bautizada es aver sido mora hasta aora y aver hecho vida de mora

preguntada que diga y particularmente declare en que ha sido mora y hecho vida de mora dixo que en hablar algaravia y en el vestir al uso de moros y en nombrar a los demas del pueblo de moros

preguntada si ha hecho otras mas ceremonias de mora mas de las que tiene confessadas dixo que no

preguntada que diga si las ceremonias de mora que ha confessado haver hecho las ha hecho con intencion y creencia de mora pensando salvarse en la secta de mahoma dixo que esta ha hecho las cosas y ceremonias de mora que tiene confessadas con intencion y voluntad de mora y esta ha tenido hasta oy y en respeto de salvarse dixo que sera lo que dios quisiere

preguntada de aqui adelante que es lo que quiere ser cristiana o mora dixo que sera lo que dios y su señoria mandaren fuele dicho que dios nuestro senor quiere que todos se salven y yo el inquisidor tambien querria que ella se salvasse y que sepa que ninguno se puede salvar sino en la ley de los cristianos por tanto que diga y declare si quiere ser cristiana y salvarse porque se le concedera el perdon y gracia que esta prometida a los nuevos convertidos del lugar de Benimodol y si ella no promete de ser cristiana no se le dara el perdon y gracia dixo que lo que dios quisiere sera y no se pudo sacar della otra cosa fuele dicho si quiere aprender las oraciones de cristiana dixo que hara lo que dios quisiere

fuele dicho que diga si o no quiere ser cristiana dixo que lo que dios quisiere y no se le pudo sacar otra cosa por muchas preguntas que se le hizieren mediante noffre ervas interprete 